
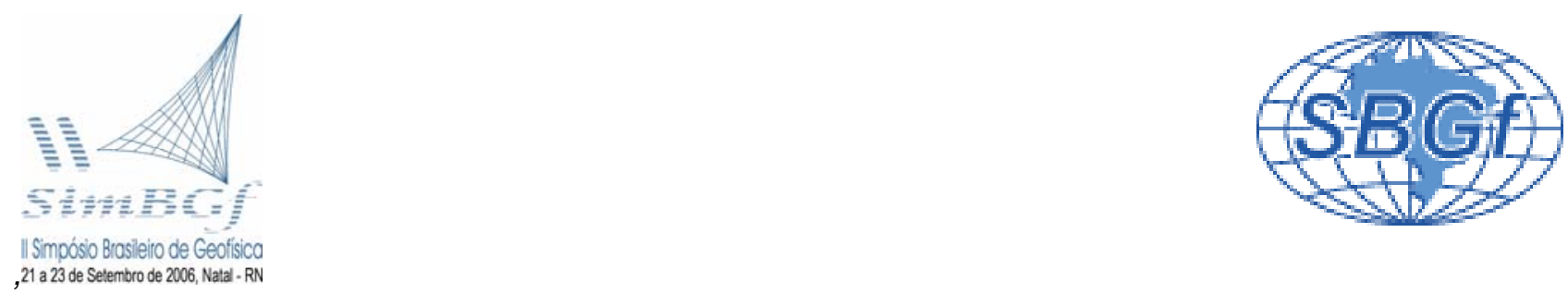

\title{
Especificação digital de cores com o auxílio de scanner e computador e classificação quantitativa de rocha ornamentais com objetivo de controle de qualidade de alta precisão por meio de parâmetros de cores físicas
}

\author{
Akihisa Motoki, DMPI/UERJ e-mail: rochasornamentais@yahoo.com.br \\ Lóris Lodir Zucco, CTUR/UFRRJ \\ Thais Vargas, DMPI/UERJ \\ Susanna Eleonora Sichel, LAGEMAE/UFF \\ José Luiz Peixoto Neves, DMPI/UERJ \\ José Ribeiro Aires, ABAST/PETROBRÁS
}

\section{Resumo}

Foi desenvolvido o método para especificação quantitativa de cores com o auxílio de tecnologia digital utilizando-se scanner e computador, com objetivo especial de aplicação para classificação de rochas ornamentais. A imagem da rocha ornamental é capturada por scanner em resolução óptica de 600 dpi no modo true colour. Para amenizar o efeito de ruído de pixels, a resolução é moderada em 300 ou 150 dpi. O software Wilbur analisa a cor da imagem e determina os parâmetros cromáticos, tais como RGB, HSB e XYZ. Para classificação das rochas ornamentais, o sistema HSB é adequado. Este técnica possibilita o controle de qualidade das rochas naturais em alta precisão, podendo elevar a competitividade no mercado de material nobre de construção.

\section{Introdução}

As industries de rochas ornamentais do Brasil está em notável crescimento desde o início do século XXI, tanto na extração de blocos em pedreiras quanto na produção de produtos acabados em fábricas. Sobretudo, a exportação dos produtos finais, isto é, placas polidas de rochas com corte dimensionado para revestimento de parede e pavimentação do piso, está em rápido crescimento, conquistando o mercado dos países industrialmente desenvolvidos. Este sucesso é creditado na introdução do maquinário da tecnologia de ponta, o que viabilizou alto nível de controle de qualidade (Motoki et al., 2005a).

Ao mesmo tempo, as indústrias de rochas ornamentais foram obrigadas a encarar na rigorosa concorrência com outros materiais, tal como cerâmica. Sendo diferente dos produtos artificiais, rochas ornamentais não têm homogeneidade perfeita, havendo a heterogeneidade natural no seu aspecto visual. Portanto, as análises das características visuais e sua medida quantitativa, especialmente de cores, são importantes para viabilizar o controle de qualidade de alto nível (Campello, 2006).
Certas rochas ornamentais do Brasil têm cores relevantes, tais como de azul (Granito Azul Bahia, Granito Azul Macaúba, Granito Cinza Azul Guanabara), preta (Granito Preto Tijuca, Granito Preto São Gabriel, Granito Preto São Benedito), vermelha (Granito Vermelho Itu, Granito Vermelho Capão Bonito), marrom (Granito Marrom Caldas, Granito Café Imperial) e cinza oscilatória (Granito Cinza Ás de Paus). Estas rochas são de alto valor comercial.

Entretanto, o controle de qualidade de cor das rochas por meios objetivos e físicos era praticamente nulo, sendo classificadas por meios subjetivos. Durante o século $X X$, não havia o método para medir as cores em parâmetros numéricos. Motoki et al. (2000) apresentou o primeiro método para a medida quantitativa de cores das rochas ornamentais a partir da imagem digital. Este método tinha eficiência suficiente no laboratório, entretanto não era prática devido ao exaustante trabalho para uma análise. Motoki et al. (2003a; b) desenvolveram o protótipo de software original Wilbur para análise quantitativa de cores e, os resultados preliminares foram apresentados na revista industrial da Itália e revista científica do Japão. Motoki et al. (2005b) aplicou esta técnica para análise de cor do Mármore Bege Bahia, Motoki et al. (2005c), para Basalto da Serra Gaúcha. Entretanto, até o presente, não havia trabalhos completos que apresenta esta técnica em idioma português. Em paralelo, (Campello \& Costa, 2003; Campello et al., 2005a; b; Campello, 2006) desenvolveram métodos para medida quantitativa de cores utilizando-se o software Quantikov.

Este trabalho apresenta o método para medida quantitativa de cores com o auxílio de scanner, computador e software Wilbur e, sua aplicação em prol de estabelecimento do padrão de classificação quantitativa de rochas ornamentais por meios de cores físicas.

\section{Metodologia}

A medida quantitativa digital de cores em rocha total e em cada mineral é realizada a partir de imagem digital de placa polida de rocha ornamental, denominada "chapa 
polida" ou "dimension stone slab". A amostra é submetida a scanner e sua imagem digital é capturada de modo true colour, de 17 milhões de cores. A resolução óptica da captura é 600 dpi. Para manter a informação óptica completa, a imagem é gravada no formato BMP. Os formatos JPG e GIF não podem ser utilizados devido à perda parcial da informação original.

A imagem capturada da rocha é submetida ao processo preparativo da análise com o auxílio de um software gráfico comercial, tal como Adobe Photoshop ${ }^{\mathrm{TM}}$. A resolução óptica da imagem original é moderada em 300, 150 ou 75 dpi. Esta moderação tem como objetivo de amenizar os efeitos de ruídos ópticos causados pela lâmpada fluorescente de scanner.

Sendo diferente do Quantikov, o Wilbur, que foi desenvolvido pelos autores, permite a seleção da área a ser analisada. Desta forma, o operador humano pode excluir da análise as manchas, riscos, buracos e outros fatores indesejados presentes na superfície da rocha polida. A área a ser analisada é indicada pelo arquivo de filtro em formato BMP, denominado selection filter (Motoki et al., 2005b). Utilizando a mesma função, pode-se analisar também a cor de um mineral específico.

O Wilbur analisa apenas os pixels das áreas marcadas no selection filter e calcula os parâmetros estatísticos dos sistemas cromáticos de RGB, HSB, XYZ e $x y z$.

\section{Classificação de rochas ornamentais por parâmetro S}

A avaliação das cores de rochas ornamentais é realizada por meio do sistema cromático HSB (hue, saturation, brightness). O parâmetro $\mathrm{S}$, saturation, das rochas ornamentais é baixo em comparação com outros materiais, tais como cores de animais, plantas e objetos artificiais (Tabela 1).

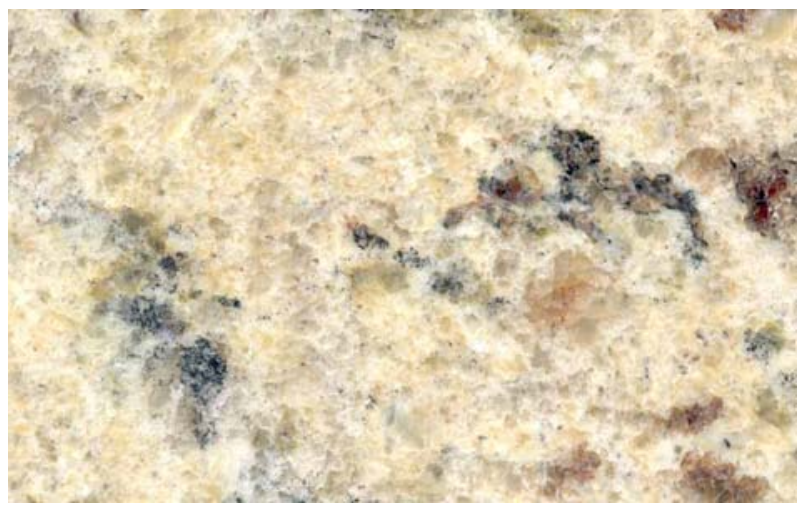

Figura 1. Granito Amarelo Icaraí, uma rocha de cor amarela relevante $(\mathrm{H}=42, \mathrm{~S}=14.8)$. A largura horizontal da imagem corresponde a $8 \mathrm{~cm}$.

Este parâmetro representa nitidez da cor e, portanto pode-se dizer que as cores de rochas ornamentais são geralmente pouco nítidas. Como por exemplo, o vermelho considerado como destacado de Granito Vermelho Itu, Granito Vermelho Capão Bonito e Granito Vermelho

II Simpósio Brasileiro da SBGf, ^
Bragança Paulista tem o S em torno de 15 (Motoki et al., 2003a; b). Este valor é alto como uma rocha ornamental, porém é baixo em comparação com as cores de

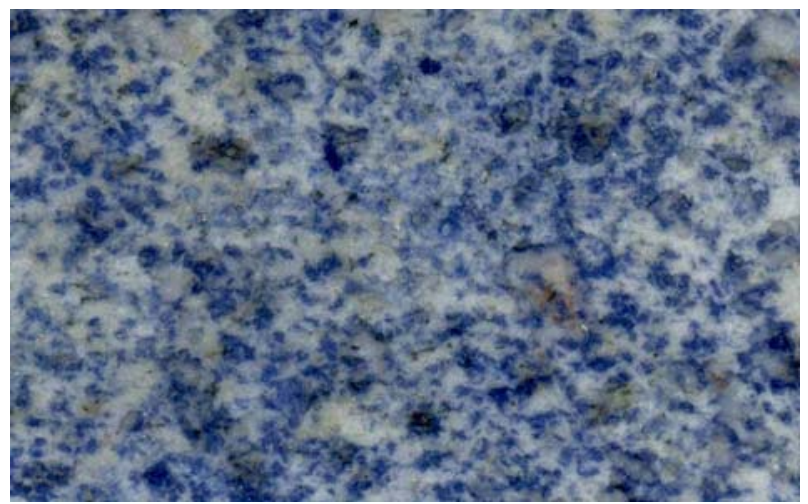

Figura 2. Granito Azul Bahia de variedade de luxo, com alto teor de sodalita e relevante cor azul $(\mathrm{H}=210, \mathrm{~S}=13.9)$. A largura horizontal da imagem corresponde a $8 \mathrm{~cm}$.

cerâmicas, sendo comparável com o vermelho acinzentado de tijolo. O parâmetro $S$ é variável desde o baixo valor de rochas de cor cinza, em torno de 3 , até as rochas vermelhas acima citadas, superior a 15.

Além das rochas vermelhas, certas rochas graníticas de cor amarela, tais como Granito Giallo Florence, Granito Amarelo Icaraí (Figura 1) e Granito Ouro Velho também têm elevado parâmetro $S$, sendo respectivamente 14.4 , 14.8 e 15.9.

O Granito Azul Bahia (Figura 2) é famoso devido à sua cor azul considerada como altamente destacada. Esta rocha tem uma grande variedade de cor azul, ou seja teor de sodalita, com o S variando de 6.3 a 13.9 (Figura 1). Este parâmetro tem influência direta do valor comercial desta rocha. Apesar da fama, a maioria das variedades desta rocha tem o S inferior a 8.0. Neste sentido, a cor amarela clara do Mármore Bege Bahia, com o S em torno de 12 , é mais relevante.

A maioria das rochas ornamentais coloridas tem cores menos destacadas do que as rochas acima citadas. O

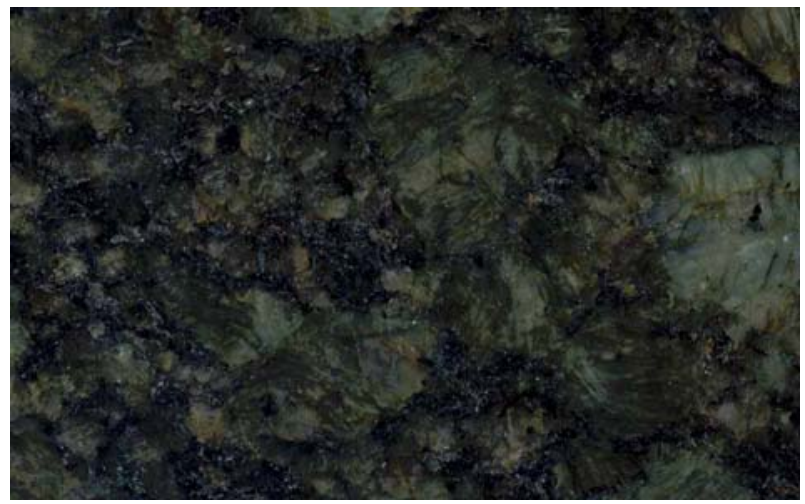

Figura 3. Granito Verde Pavão, uma rocha ornamental charnockítica representativa do Brasil, de cor verde escura $(\mathrm{H}=135, \mathrm{~S}=4.8, \mathrm{~B}=9.1)$. Notam-se baixos valores dos parâmetros S e B. A largura horizontal da imagem corresponde a $8 \mathrm{~cm}$. 
charnockito Granito Verde Pavão (Figura 3) é valorizada pela sua cor verde, entretanto o $\mathrm{S}$ é baixo, sendo 4.8. O Granito Café Imperial, de cor marrom, tem o $\mathrm{S}$ variando de em torno de 4. Portanto, as cores destas rochas são, de fato, pouco relevante.

As rochas ornamentais popularmente chamadas de granito cinza possuem o $\mathrm{S}$ muito baixo, sendo que o Granito Cinza Ás de Paus (Figura 4) e Granito Cinza Corumbá possuem o parâmetro $\mathrm{S}$ nas faixas respectivas de 3.0 3.3 e 3.6 3.9. Os granitos pretos, Granito Preto São Gabriel e Granito Preto São Benedito, têm o S respectivamente 2.7 e 3.6, e os granitos brancos, Granito Branco Ceará, Granito Branco Fortaleza, Granito Inada possuem o $\mathrm{S}$ respectivamente $1.9,3.7$ e 3.1 .
As observações acima citadas permitem a classificas rochas ornamentais pelo parâmetro $S$ em seguintes 5 categorias: rocha de escala de cinza (GS, greyscale rock), $\mathrm{S}<4$; cor ligeira (SC, slight colour), $4<\mathrm{S}<7$; cor baixa ( $L C$, low colour); $7<\mathrm{S}<10$; cor média $(M C$, middle colour), $10<\mathrm{S}<13$; cor relevante (HC, high colour), $\mathrm{S}>13$ (Figura 6).

Essas categorias foram propostas por Motoki et al. (2003a; b), entretanto os limites divisores entre as categorias do presente trabalho têm o $\mathrm{S}$ mais elevado. Este fenômeno é devido à evolução de hardware, sobretudo de sensor de scanner.

Para as rochas de escala de cinza, o parâmetro $\mathrm{H}$ é pouco significativo. Entretanto, o parâmetro B, brightness,

Tabela 1 - Cores medidas pelo presente método em HSB de principais rochas ornamentais.

\begin{tabular}{|c|c|c|c|c|c|}
\hline Nome Comercial & Nome científico & Cor & $\mathrm{H}$ & $\mathrm{S}$ & $\mathrm{B}$ \\
\hline Granito Azul Bahia 1 & sodalita sienito & Azul & 139.6 & 6.8 & 51.6 \\
\hline Granito Azul Bahia 2 & sodalita sienito & Azul & 199.0 & 8.2 & 44.7 \\
\hline Granito Azul Bahia 3 & sodalita sienito & Azul & 210.0 & 13.9 & 51.8 \\
\hline Granito Azul Bahia 4 & sodalita sienito & Azul & 184.5 & 6.3 & 43.0 \\
\hline Granito Azul Bahia 5 & sodalita sienito & Azul & 192.5 & 7.6 & 49.4 \\
\hline Mármore Bege Bahia 1 & calcário & Amarelo claro & 37.7 & 11.5 & 90.7 \\
\hline Mármore Bege Bahia 2 & calcário & Amarelo claro & 37.6 & 12.8 & 92.4 \\
\hline Granito Amarelo Icarai & gnaisse & Amarelo claro & 42.0 & 14.8 & 85.0 \\
\hline Granito GialloFlorence & granito & Amarelo & 50.2 & 14.4 & 56.8 \\
\hline Granito Ouro Velho & granito & Amarelo & 46.6 & 15.9 & 64.4 \\
\hline Granito Verde Pavão & charnockito & verde escuro & 135.8 & 4.8 & 9.1 \\
\hline Granito Café Imperial 1 & álcali sienito & Marrom & 87.0 & 3.9 & 19.2 \\
\hline Granito Café Imperial 2 & álcali sienito & Marrom & 352.1 & 4.3 & 9.8 \\
\hline Granito Café Imperial 3 & álcali sienito & Marrom & 80.4 & 5.0 & 20.1 \\
\hline Mesquita & nefelina sienito & Marrom & 90.9 & 3.3 & 60.1 \\
\hline Ardosia Micapal Azul & ardósia & azul escuro & 198.5 & 5.9 & 39.9 \\
\hline Ardosia Micapal Verde & ardósia & verde escuro & 163.2 & 5.7 & 57.2 \\
\hline Ardosia Micapal Cinza & ardósia & cinza escura & 99.1 & 0.9 & 47.1 \\
\hline Granito Preto São Gabriel & diorito & Preto & 164.7 & 2.7 & 11.6 \\
\hline Granito Preto São Benedito & diorito & Preto & 341.8 & 3.6 & 12.3 \\
\hline Basalto da Serra Gaúcha & riolito & cinza escura & 78.9 & 3.8 & 46.9 \\
\hline Granito Cinza Ás de Paus 1 & nefelina sienito & Cinza & 106.5 & 3.3 & 62.4 \\
\hline Granito Cinza Ás de Paus 2 & nefelina sienito & Cinza & 131.7 & 3.0 & 64.6 \\
\hline Granito Cinza Corumba 1 & granito & Cinza & 152.8 & 3.6 & 67.3 \\
\hline Granito Cinza Corumba 2 & granito & Cinza & 142.5 & 3.7 & 67.9 \\
\hline Granito Cinza Corumba 3 & granito & Cinza & 145.8 & 3.9 & 66.9 \\
\hline Bandai Granite & granodiorito & Cinza & 163.5 & 4.7 & 54.4 \\
\hline Inada Granite & granito & cinza clara & 113.3 & 3.1 & 77.1 \\
\hline Granito Branco Fortaleza & Granito & cinza clara & 109.0 & 3.7 & 80.3 \\
\hline Granito Branco Ceará & granito pórfiro ? & Branco & 93.5 & 1.9 & 96.4 \\
\hline
\end{tabular}


que representa a clareza da cor, é importante. Desta

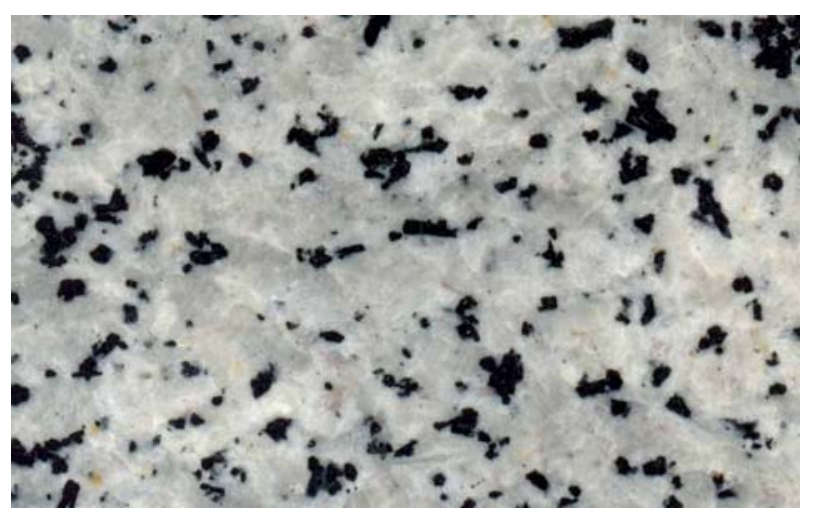

Figura 4. Granito Cinza Ás de Paus. Uma rocha ornamental rara no mundo composto de nefelina sienito, de cor cinza clara $(S=3.3, B=62.4)$. A largura horizontal da imagem corresponde a $8 \mathrm{~cm}$.

forma, as rochas de escala de cinza e uma parte das rochas de cor ligeira são subclassificadas pelo parâmetro B.

As rochas ornamentais chamadas de granito preto (de fato esses são quartzo diorito e diorito), tais como Granito Preto São Gabriel ( $\mathrm{B}=11.6)$ e Granito Preto São Benedito $(B=12.3)$ têm o $B$ baixo, sendo geralmente inferior a 20. No caso das rochas com o B muito baixo, a cor sentida por olho humano é preta, mesmo com o S pouco elevado.

Certas rochas de aplicação geral com superfície não polida, tais como Basalto da Serra Gaúcha (riolito) e determinado tipo de ardósia cinza, têm cor cinza escura com o parâmetro B em torno de 45.

Rochas graníticas de uso popular têm cor cinza relativamente clara, como Granito Cinza Andorinha e Granito Cinza Corumbá, com o parâmetro B em torno de 65. O Granito Cinza ás de Paus também tem o parâmetro $B$ na mesma faixa. Algumas rochas nobres de cor cinza clara, chamadas eventualmente de granito branco, tais como Granito Branco Fortaleza e Inada Granite, têm o B ao redor de 75 .

As rochas tipicamente brancas, como Mármore Branco Cintilante e Granito Branco Ceará (Figura 5), têm o parâmetro B superior a 85.

Essas observações permitem a subclassificação das rochas de escala de cinza pelo parâmetro $B$ em seguintes 4 subcategorias: rocha "preta" ( $B$, black rock), $0<B<25$; "cinza escura" (DG, dark grey), $25<\mathrm{B}<55$; "cinza clara" (LG, light grey), 55<B<85; "branca" (W, white), de B>85 (Figura 6). Os limites entre as subcategorias são mesmos de Motoki et al. (2003a; b).

\section{Classificação de rochas ornamentais por parâmetro H}

Ao contrário dos casos de rochas de escala de cinza, o parâmetro $\mathrm{H}$, hue, é importante para rochas de cor relevante e cor média, até uma parte de rochas de cor baixa. Este parâmetro representa a tonalidade da cor, tais

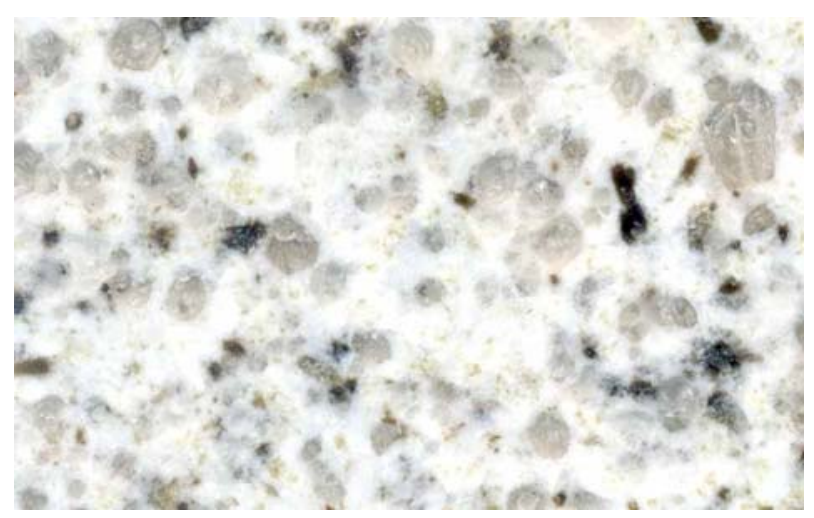

Figura 5. Granito Branco Ceará. A rocha ornamental com forte característica da cor branca $(S=1.9, B=91.4)$. Notamse muito alto $B$ e baixo $S$. A largura horizontal da imagem corresponde a $8 \mathrm{~cm}$.

como vermelho, amarelo, verde e azul. Motoki et al. (2003a; b) propuseram provisoriamente 3 subcategorias conforme o parâmetro $\mathrm{H}$ : vermelho, $0<\mathrm{H}<20$; laranja, $20<\mathrm{S}<40$; amarelo, $40<\mathrm{H}<60$.

De acordo com o aprimoramento de hardware e aquisição recentes de ampla variedade de amostras de rochas

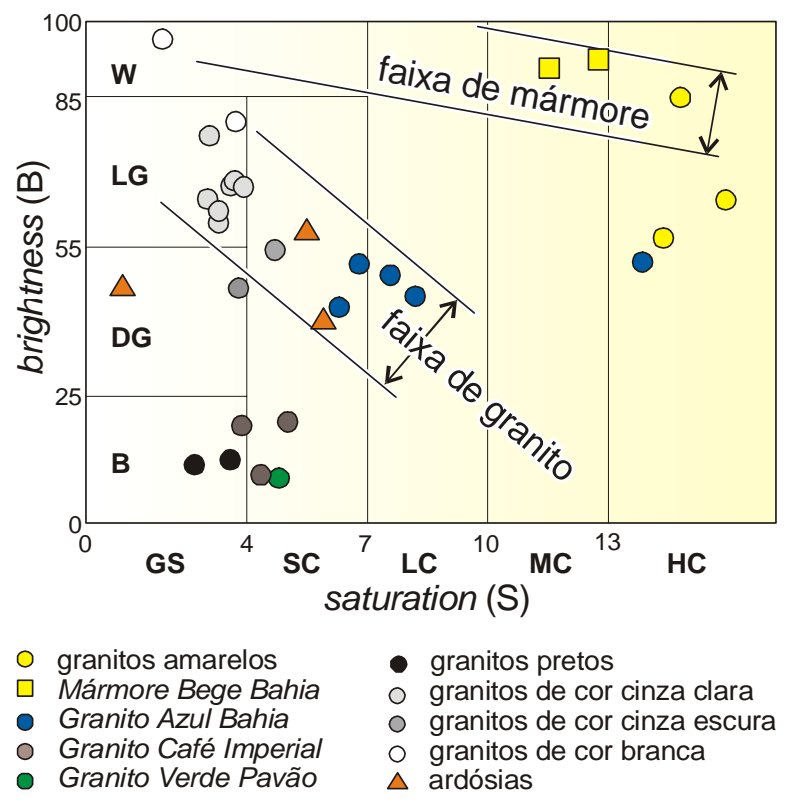

Figura 6. Diagrama saturation-brightness (S-D diagram) para classificação de rochas ornamentais por meio de cores físicas. As categorias conforme o $\mathrm{S}$ são: GS - escala de cinza, S<4; SC - cor ligeira, $4<\mathrm{S}<7$; LC- cor baixa, $7<\mathrm{S}<10$; MC - cor média, $10<S<13$; cor relevante, $S>13$. As subcategorias conforme o B são: B - preta, B<25; DG - cinza escura, $25<B<55$; LG - cinza clara, 55<B<85; W - branca, $\mathrm{B}>85$. 
ornamentais, especialmente de Granito Azul Bahia e ardósia colorida, o presente trabalho propõe a subclassificação complementar, porém ainda de caráter provisório, em seguintes 6 subcategorias: vermelho (red), $350>\mathrm{H}<360, \quad 0<\mathrm{H}<20$; laranja (orange); $20<\mathrm{H}<40$; amarelo (yellow), $40<\mathrm{H}<60$; verde (green), $60<\mathrm{H}<180$; azul (blue), $180<\mathrm{H}<270$; roxo (purple), $270<\mathrm{H}<350$.

O diagrama saturation-hue (S-H diagram; Figura 7) visualiza esta classificação. Neste momento, não há ainda dados de Granito Vermelho Capão Bonito (álcali granito), Granito Vermelho Bragança Paulista (álcali granito) e Granito Roxo Gaúcho (álcali sienito) pelo novo sistema de hardware. Uma amostra de Granito Azul Bahia que se situa na área verde tem alto teor de epidoto, além de sodalita.

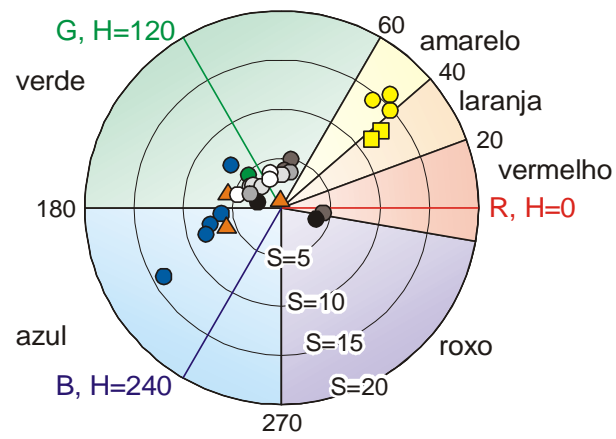

- granitos amarelos

$\square$ Mármore Bege Bahia

- Granito Azul Bahia

- Granito Café Imperial

- Granito Verde Pavão

- granitos pretos

- granitos de cor cinza clara

- granitos de cor cinza escura

- granitos de cor branca

$\triangle$ ardósias

Figura 7. Diagrama saturation-hue (S-H diagram) para classificação de rochas de cores média e relevante. A definição dos limites entre as subcategorias ainda está em desenvolvimento.

\section{Discussões}

A classificação pelo parâmetro $S$ apresentada no presente trabalho tem limites divisores diferentes de Motoki et al. (2003a; b). O scanner utilizado para esses trabalhos foi Genius ${ }^{\mathrm{TM}}$ Color Vivid II $^{\mathrm{TM}}$, que é um dos primeiros modelos fabricado no final do século passado. Os scanners daquela época não tinham fidelidade na reprodução de cores suficientemente alta para a análise quantitativa digital. Sobretudo no caso das rochas com o $\mathrm{S}$ inferior a 7.0, o problema foi sério.

As cores das imagens capturadas eram significativamente menos nítidas e, portanto o $\mathrm{S}$ medido tendia a apresentar um valor inferior. Além disso, houve o problema de descalibração na sensibilidade dos canais $\mathrm{R}, \mathrm{G}$ e B. Portanto, o efeito dos problemas relativos ao baixo parâmetro $\mathrm{S}$, chamados de "charnockite problem" (Motoki \& Zucco, 2005), era crítico.
A tecnologia de reprodução de cores foi aprimorada notavelmente nos últimos anos. Hoje em dia, até mesmo os modelos populares possuem fidelidade de cores relativamente elevada. Portanto, o charnockite problem foi amenizado, aprimorando a confiabilidade da medida quantitativa de cores. Este fator viabilizou a definição detalhada das subcategorias relativas ao parâmetro $\mathrm{H}$.

A Figura 7 apresenta que as rochas de cor cinza e branca não estão espalhadas homogeneamente em torno do ponto $S=0$, mas são deslocadas ligeiramente à direção da cor verde. Esta tendência pode ser reconhecida a olho nu em certos espécimes, tal como Granito Branco Ceará. Entretanto, poderia ser atribuída também à descalibração do scanner. No futuro próximo, a evolução do hardware viabilizará a calibração de cor de scanner via software, utilizando-se a chapa padrão de cores (colour chart) de Kodak $^{T M}$.

O método desenvolvido até o presente foi destinado à análises de cor física de rochas ornamentais conforme o padrão CIE1931, de Comission Internacionale de L'Éclariage. Quantitativamente, este sistema e os relacionados, inclusive o sistema HSB, não é proporcional à sensibilidade de olhos humanos. O sistema cromático conforme a sensibilidade mental ainda não foi estabelecido, o sistema $L^{*} a^{*} b^{\star}$ do padrão CIE1976 é mais próximo. Para avaliação das cores de rochas ornamentais, deseja-se o desenvolvimento no futuro próximo o método com base no sistema cromático $L^{*} a b^{*}$.

Esta tecnologia estenderá suas aplicabilidades em vários outros campos de geociências. Entre esses, a análise modal automática dos minerais constituintes de rochas (Motoki et al. 2006) e a estimativa simples do teor de hidrocarboneto das rochas geradoras de petróleo são mais destacadas. A fidelidade da reprodução de escala de cinza é alta e este fator facilitará a aplicação da presente técnica à avaliação das rochas geradoras do petróleo.

\section{Conclusões}

1) A cor das rochas ornamentais é analisada quantitativamente a partir de imagem digital de scanner com o auxílio de computador e software original Wilbur.

2) A captura digital de imagem por scanner é realizada em 600 dpi no modo true colour. A imagem é gravada em formato BMP. Essa imagem é moderada em resolução óptica de 300 dpi ou 150 dpi.

3) O sistema cromático HSB é adequado para a análise e classificação de rochas ornamentais pela cor física.

4) As rochas são classificadas, em primeiro, pelo $S$ em 5 categorias: escala de cinza, $\mathrm{S}<4$; ligeira, $4.0<\mathrm{S}<7.0$; baixa, $10.0<\mathrm{S}<7.0$; média, $13.0<\mathrm{S}<10.0$; relevante S $>13$.

5) Rochas de escala de cinza são subclassificadas pelo $B$ em 4 subcategorias: preto, $B<25$; cinza escura, $25<B<55$; cinza clara, 55<B<85; branco, B>85.6;

6) Rochas de cor relevante são subclassificadas pelo $\mathrm{H}$ em 6 subcategorias: vermelho, $350<\mathrm{H}<360,0<\mathrm{H}<20$; 
laranja; $20<\mathrm{H}<40 ; \quad$ amarelo, $40<\mathrm{H}<60$; verde, $60<\mathrm{H}<180$; azul, $180<\mathrm{H}<270$; roxo, $270<\mathrm{H}<350$.

\section{Agradecimentos}

Os autores agradecem aos alunos Giannis Hans Petrakis e Rodrigo Soares, da Universidade do Estado do Rio de Janeiro, pela dedicação aos trabalhos em prol do desenvolvimento da presente técnica e ao Sr. Sócrates Giannis Petrakis de Partenon Mármores, Rio de Janeiro, pelo fornecimento das amostras de rochas ornamentais.

\section{Referências}

Campello, M.S., Costa, A.G. 2003. Desenvolvimento de metodologia para o emprego de Quantikov - software analisador de imagens, na determinação da análise modal tipos pétreos com valor comercial. Bol. Res. Simpósio Regional de Geologia de Minas Gerais, Belho Horizonte (MG), CD.

Campello, M.S., Bruno, R., Costa,A.G., Moura, A.C. 2005a. Proposta de classificação das rochas ornamentais com base em parâmetros estéticos. Ann. $1^{\text {st }}$ International Congress of Dimension Stones (ICIRO), Guarapari (ES), CD.

Campello, M.S., Costa, A.G., Mouora, A.C., Freitas, C.R., Pinto, L.M. 2005b. Técnica de processamento digital de imagens para correlação entre a forma dos grãos e característica tecnológica de granitos ornamentais. Boletim de Resumos de $9^{\circ}$ Simpósio Regional de Geologia do Sudeste, $13^{\circ}$ Simpósio Regional de Geologia de Minas Gerais, Niterói (RJ), CD.

Campello, M.S. 2006. Técnicas de processamento digital de imagens com aplicação no setor das rochas ornamentais. Tese de doutorado. Instituto de Geociências da Universidade Federal de Minas Gerais, Belo Horizonte (MG), 165p. (inédito)

Motoki, A., Neves, J.L.P., Vargas, T. 2005a. Digital specification of whole-rock and spot colours of Mármore Bege Bahia, a Brazilian light yellow limestone of breccia texture. Ann. $1^{\text {st }}$ International Congress of Dimension Stones, Guarapari (ES), CD, 6p.

Motoki, A., Neves, J.L.P., Vargas, T. 2005b. Quantitative colour analyses using digital specification technique for Mármore Bege Bahia, a representative Brazilian ornamental limestone of breccia-like texture. Revista Escola de Minas, Ouro Preto (MG), 58-2: 113-120.

Motoki, A., Zucco, L.L., Freitas, A. 2005c. Ornamental and artistic applications of Basalt, an attractive variation of porphyry of south-most Brazil, with special attention of its colour variation. Ann. $1^{\text {st }}$ International Congress of Dimension Stones, Guarapari (ES), CD, $6 \mathrm{p}$.

Motoki, A., Zucco, L.L. Digital 2005. Colour specification for ornamental rocks using image scanner: its technical challenge, on-going development, further innovation, and application. Ann. $1^{\text {st }}$ International Congress of Dimension Stones (ICIRO), Guarapari (ES), CD, 6p.

Motoki A., Vargas T., Peixoto J.L.N. 2000. Análise quantitativa das cores de feldspato alcalino, nefelina e sodalita em rochas ornamentais. Mineração Metarulgia. 554-06: 14-30.

Motoki, A., Vargas, T., Neves, J.L.P., Zucco, L.L. 2003a. Quantitative analyses of rock and mineral surface color for ornamental and semi-ornamental rocks using image scanner. Japanese Magazine of Mineralogical and Petrological Sciences, 32-1: 12-22.

Motoki, A., Vargas, T., Neves, J.L.P., Zucco, L.L. 2003b. Pietre naturali classificate per colori, una técnica de misurazione quantitativa del colore per rocce ornamentali e semi-ornamentali, utilizzando scanner e computer. L'Informatore del Marmistas, 493: 6-16. 